\title{
Spontaneous Subgaleal Hematoma in a Sickle Cell Disease Patient: A Case Report
}

\author{
Moayad Majed Alqurashi, g, Omar Muntaser Raslan ${ }^{\text {b, c, }}$ \\ Giamal Edin Gmatid, e, f
}

\begin{abstract}
Sickle cell disease (SCD) is one of the most commonly inherited conditions on the Arabian Peninsula. We report a case of a 17-yearold boy, who had previously been diagnosed with SCD and glucose6-phosphate dehydrogenase (G6PD) deficiency and recently presented to our hospital with spontaneous subgaleal hematoma (SGH), which was managed conservatively. We also present a literature review on the topic of spontaneous intra- and/or extra-cranial bleeds.
\end{abstract}

Keywords: Subgaleal hematoma; Extradural hematoma; Sickle cell disease; Saudi Arabia

\section{Introduction}

Sickle cell disease (SCD) is one of the most commonly inherited autosomal recessive conditions in people of African, Indian, Arabian Peninsula, and Mediterranean descent [1, 2]. The oxygen-carrying hemoglobin molecules of red blood cells are affected in this hemoglobinopathy, predisposing the blood cells to a sickle-shape that may occlude small arteries and cause tissue hypoxia and micro/macro infarcts $[1,2]$. Five to seven percent of the worldwide population is estimated to have hemoglobinopathies with Africa having the highest prevalence of SCD $[3,4]$. The prevalence of SCD in Saudi Arabia is higher than that in other countries, especially in the Eastern

Manuscript submitted February 2, 2020, accepted February 21, 2020

aDepartment of Medicine, King Abdulaziz Medical City, Riyadh, Saudi Arabia bHematology Division, London Health Sciences Centre, London, Ontario, Canada

${ }^{\mathrm{c}}$ Department of Medicine, University of Jeddah, Jeddah, Saudi Arabia

dDivision of Adult Hematology and HSCT, Department of Oncology, King Abdulaziz Medical City, Riyadh, Saudi Arabia

${ }^{\mathrm{e} C}$ College of Medicine, King Saud bin Abdulaziz University for Health Sciences, Riyadh, Saudi Arabia

fKing Abdullah International Medical Research Center, Riyadh, Saudi Arabia gCorresponding Author: Moayad Majed Alqurashi, Adult Infectious Diseases Division, Department of Medicine, Makkah Street, Prince Sultan Military Medical City, P.O. BOX 7897, Riyadh 11159, Saudi Arabia.

Email: moayad.alqurashi@hotmail.com

doi: https://doi.org/10.14740/jmc3433 and Southern regions of the country with 145 and 24 cases per 10,000 people, respectively; however, the disease is decreasing [3]. Moreover, around $2 \%$ to $27 \%$ of the Saudi population is considered to carry the disease depending on the area and the high rate of consanguineous marriages [3].

Patients with SCD suffer from multiple types of complications that affect all organs and range from recurrent pain to end-organ damage and failure and recurrent infections [3]. In this report, we describe a patient with SCD who presented with spontaneous subgaleal hematoma ( $\mathrm{SGH}$ ) and we also provide a brief review of the literature concerning this topic.

\section{Case Report}

A 17-year-old Saudi boy from the Southern region, who had previously been diagnosed with SCD and glucose-6-phosphate dehydrogenase (G6PD) deficiency, presented to the emergency department (ER) after he had scalp pain with increasing spontaneous swelling that started 3 days earlier. He had no history of trauma, altered mental status, vision changes, or diplopia but presented with left-side facial swelling mainly at the site of the parotid gland and the mandible that had started 5 days earlier. Presently, he is compliant on hydroxyurea and folic acid tablets once daily with no reports of SCD complications or vaso-occlusive crisis (VOC) attacks (last VOC episode that required hospitalization was around 2 years prior to the present presentation). He had a similar episode of a swelling on the head 1 year ago that resolved spontaneously without seeking medical advice. He also underwent laparoscopic cholecystectomy due to cholecystitis 3 years prior to the present presentation.

Physical examination revealed only mild tender bilateral swelling $(5 \times 5 \mathrm{~cm})$ over the parietal areas of the scalp and at the left parotid gland site $(3 \times 3 \mathrm{~cm})$. The scalp swelling was fluid-filled as shown by ultrasound, while other bony face and skull prominences were not tender. The neurological examination was unremarkable and showed intact motor, sensory, and cranial nerves. Visual field, extraocular movements, and visual acuity measurements were normal.

His initial laboratory investigations in the ER were unremarkable. He had a hemoglobin of $10.7 \mathrm{~g} / \mathrm{dL}$. His latest hemoglobin electrophoresis showed a $68.3 \%$ concentration of hemoglobin S. Computed tomography of his face and skull showed bilateral frontal and parietal SGHs without any intracranial abnormalities (Fig. 1), and the underlying skull showed 


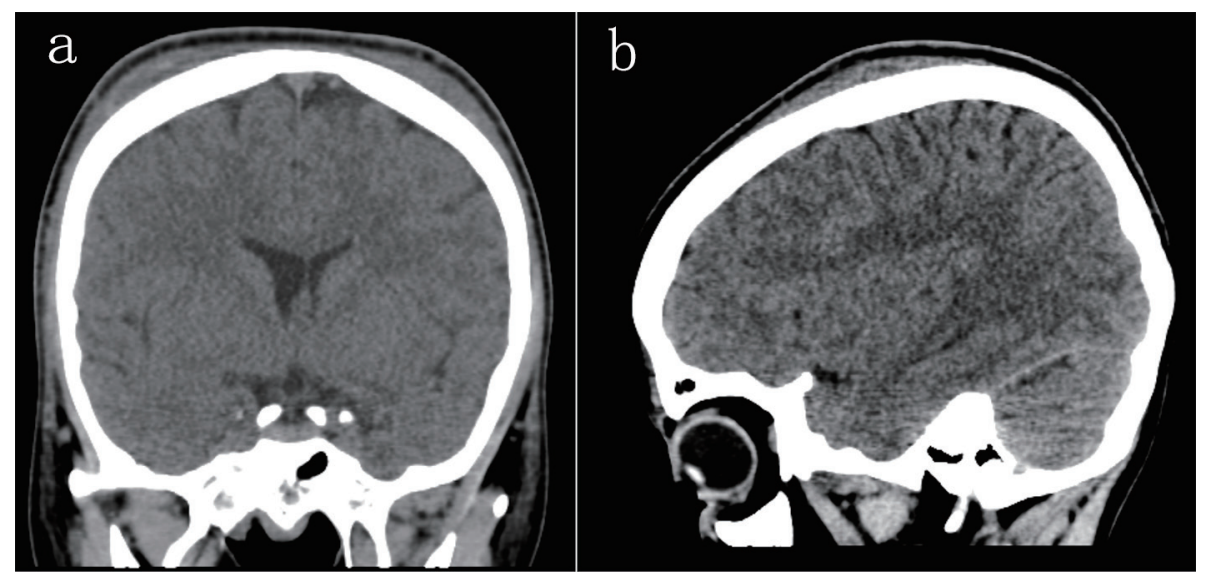

Figure 1. Sagittal and coronal views of a brain computed tomography without contrast showing bilateral frontal and parietal subgaleal hematomas without any signs of bone infarctions.

no signs of erosion, fractures, or signs of ischemia.

He was managed conservatively with simple analgesia and educated on the possibility of spontaneous intracranial bleeding and the need for urgent hospital visit in case of any neurological deficits suggestive of intracranial bleeding development. His symptoms resolved spontaneously in few days. After 6 months, he was seen in the outpatient clinic for regular follow-up and he denied any recurrence of similar symptoms or other SCD complications.

\section{Discussion}

Silent and acute parenchymal infarctions and moyamoya vasculopathy comprise most neurological complications of SCD [1]. Two-third of the central nervous system complications are ischemic; intracerebral hemorrhages are less common [2]. Subarachnoid or spontaneous extradural hematomas are even less common than the other two complications [2]. These central nervous system (CNS) complications can reach up to $6.9 \%$ in children with SCD compared to $0.5 \%$ in healthy children [1]. Spontaneous epidural hematomas (EDHs) are rare, and a patient with such a hematoma may present with headaches or other acute neurological symptoms [1].

Although an extra-axial hemorrhage in SCD is very rare [5], reports of all types of extra- or intra-axial hemorrhages or combinations of them have been shown to be associated with SCD [6]. In adults, an SGH is usually caused by trauma and involves blood accumulation between the epicranial aponeurosis or galea aponeurotica and the periosteum because of rupture of the emissary veins [7]. SGH has been reported in SCD patients presenting with SGH in combination with spontaneous EDH [1, 2, 5, 8, 9]. Some reports of spontaneous $\mathrm{EDH}$ in association with other calvarial infarctions have been published. Most of these cases are trauma-related while other causes include infections, coagulopathies, vascular malformation, tumors, open heart surgery, hemodialysis, and systemic lupus erythromatosis $[1,5]$. The most common areas of bone infarction in SCD patients are the appendicular skeleton and spine, but subperiosteal collection mimicking an abscess at the lateral orbital wall is also a recognized complication of SCD [1].

Multiple theories on why spontaneous bleeding occurs in SCD have been proposed, including disruption of vessel walls and bone margins because of extracranial or dural periosteal elevation in cases of bone infarction, vessel rupture around the ischemic area due to inflammation or venous congestion due to insufficient venous drainage, and finally, margin disruption due to abnormal skull anatomy and proliferation in response to chronic anemia [1].

Clinically, most of the spontaneous bleeds happen shortly after an acute attack of sickle cell crisis, which could be caused by an increased demand on bone that has already suffered from anatomical disruptions and a decrease in supply and drainage that may promote further ischemia and infarction [2].

In a recent review on spontaneous hematomas in SCD, it was found that most of the patients were of the homogenous hemoglobin SS (HgbSS) genotype, and the most common location was the frontal area. Neither side appears to be more common than the other (left versus right and/or bilateral), and the majority of cases had skull infarcts that were confirmed radiologically and/or intra-operatively with around an $18.2 \%$ mortality rate [10].

Although there are reports of spontaneous SGHs in the literature, to our knowledge none of them presented with isolated spontaneous SGHs as in this case. One case was similar to our case but with EDH on one side of the head and an SGH on the other side [10]. This finding was attributed to a skull infarction, which could also have been the reason for the hematoma in our case.

\section{Conclusions}

SGH is a very rare but non-lethal complication of SCD, and it may present silently or with other intracranial bleeds. Spontaneous SGH is mostly due to bone infarction or minor head traumas, but it can also present after VOC episodes. No medical or surgical intervention is needed for SGH management. 


\section{Acknowledgments}

None to declare.

\section{Financial Disclosure}

None to declare.

\section{Conflict of Interest}

There was no conflict of interest.

\section{Informed Consent}

Not applicable since all identifiers were omitted.

\section{Author Contributions}

MMA and OMR wrote the manuscript; GEG was the treating physician and supervisor.

\section{Data Availability}

The authors declare that data supporting the findings of this study are available within the article.

\section{References}

1. Komarla R, Soares BP, Chern JJ, Milla SS. Spontane- ous epidural hematoma secondary to bone infarction in sickle cell anemia: case report. J Neurosurg Pediatr. 2018;22(1):18-21.

2. Mishra SS, Senapati SB, Gouda AK, Behera SK, Patnaik A. Spontaneous extradural and subgaleal hematoma: A rare neurosurgical crisis of sickle cell disease. Asian J Neurosurg. 2017;12(1):47-50.

3. Alotaibi MM. Sickle cell disease in Saudi Arabia: A challenge or not. J Epidemiol Glob Health. 2017;7(2):99101.

4. Madani BM, Al Raddadi R, Al Jaouni S, Omer M, Al Awa MI. Quality of life among caregivers of sickle cell disease patients: a cross sectional study. Health Qual Life Outcomes. 2018;16(1):176.

5. Serarslan Y, Aras M, Altas M, Kaya H, Urfali B. Nontraumatic spontaneous acute epidural hematoma in a patient with sickle cell disease. Neurocirugia (Astur). 2014;25(3):128-131.

6. Yogarajah M, Agu CC, Sivasambu B, Mittler MA. $\mathrm{HbSC}$ disease and spontaneous epidural hematoma with Kernohan's notch phenomena. Case Rep Hematol. 2015;2015:470873.

7. Chen CE, Liao ZZ, Lee YH, Liu CC, Tang CK, Chen YR. Subgaleal hematoma at the contralateral side of scalp trauma in an adult. J Emerg Med. 2017;53(5):e85e88.

8. Dahdaleh NS, Lindley TE, Kirby PA, Oya H, Howard MA, 3rd. A "neurosurgical crisis" of sickle cell disease. J Neurosurg Pediatr. 2009;4(6):532-535.

9. N'Dri Oka D, Tokpa A, Bah A, Derou L. Spontaneous intracranial extradural hematoma in sickle cell disease. J Neurol Surg Rep. 2015;76(1):e97-99.

10. Hettige S, Sofela A, Bassi S, Chandler C. A review of spontaneous intracranial extradural hematoma in sicklecell disease. Acta Neurochir (Wien). 2015;157(11):20252029; discussion 2029. 